\title{
Dirichlet and Neumann Boundary Value Problems for the Polyharmonic Equation in the Unit Ball
}

\author{
Valery Karachik (D)
}

check for updates

Citation: Karachik, V. Dirichlet and Neumann Boundary Value Problems for the Polyharmonic Equation in the Unit Ball. Mathematics 2021, 9, 1907. https: / / doi.org/10.3390/ math9161907

Academic Editor: Christopher Goodrich

Received: 24 June 2021

Accepted: 7 August 2021

Published: 10 August 2021

Publisher's Note: MDPI stays neutral with regard to jurisdictional claims in published maps and institutional affiliations.

Copyright: (c) 2021 by the author. Licensee MDPI, Basel, Switzerland. This article is an open access article distributed under the terms and conditions of the Creative Commons Attribution (CC BY) license (https:// creativecommons.org/licenses/by/ $4.0 /)$.
Department of Mathematical Analysis, South Ural State University, 454080 Chelyabinsk, Russia; karachik@susu.ru

\begin{abstract}
In the previous author's works, a representation of the solution of the Dirichlet boundary value problem for the biharmonic equation in terms of Green's function is found, and then it is shown that this representation for a ball can be written in the form of the well-known Almansi formula with explicitly defined harmonic components. In this paper, this idea is extended to the Dirichlet boundary value problem for the polyharmonic equation, but without invoking the Green's function. It turned out to find an explicit representation of the harmonic components of the $m$-harmonic function, which is a solution to the Dirichlet boundary value problem, in terms of $m$ solutions to the Dirichlet boundary value problems for the Laplace equation in the unit ball. Then, using this representation, an explicit formula for the harmonic components of the solution to the Neumann boundary value problem for the polyharmonic equation in the unit ball is obtained. Examples are given that illustrate all stages of constructing solutions to the problems under consideration.
\end{abstract}

Keywords: polyharmonic equation; Dirichlet problem; Neumann problem; Green's function; Almansi representation

MSC: 35J40; 31B30; 35J08

\section{Introduction}

Many works are devoted to the construction of Green's function in an explicit form for various classical boundary value problems. In [1], using the harmonic Green's function of the Dirichlet problem, the Green's functions of the Dirichlet, Neumann, and Robin biharmonic problems in a two-dimensional disk are constructed. In [2], an explicit representation of Green's function for the Robin problem for the Poisson equation is given, and in $[3,4]$ an explicit form of Green's function for the biharmonic and three-harmonic equations in the unit ball are obtained. An explicit form of the Green function in a sector for the biharmonic and triharmonic equations is given in [5,6]. The Green's function of the Neumann problem for the Poisson equation in the half-space $\mathbb{R}_{+}^{n}$ is constructed in [7], and the Green's function for the Robin problem in the disk is studied in [8]. Explicit forms of Green's function $G_{m}(x, \xi)$ of the Dirichlet problem for the polyharmonic equation [9] in the unit ball $S=\left\{x \in \mathbb{R}^{n}:|x|<1\right\}$ are constructed in various ways in [10-13], and so forth. In the paper [12], an explicit representation of Green's function $G_{m}(x, \xi)$ depending on the parity of $n$ and the positivity of $2 m-n$ is also obtained, and in the paper [13], a representation of the polynomial solution of the Dirichlet problem with polynomial boundary data and on the polynomial right-hand side, which is not easy to obtain explicitly knowing the function $G_{m}(x, \xi)$, is presented. The solvability of various Neumann-type problems and their generalizations in the unit ball for the biharmonic and polyharmonic equation are analyzed in [14-16]. In [17], for the boundary value problems for the polyharmonic equation with normal derivatives in the boundary conditions, the sufficient condition for these problems to be Fredholm is obtained, and a formula for their index is given. 
Of the recent works on the construction of Green's function for various problems, we note the papers [18-22], and on the application of Green's function to the problems of Mechanics and Physics, the works [22-26].

In the paper [27], integral representations of solutions and Green's functions of the Navier and Riquier-Neumann problems for the biharmonic equation in the unit ball are obtained. The following statement is proved in ([28] [Theorem 2]).

Theorem 1. Let $\varphi_{0} \in C^{2+\varepsilon}(\partial S), \varphi_{1} \in C^{1+\varepsilon}(\partial S)$, and $f \in C^{1}(\bar{S})$, then a solution of the Dirichlet problem for the biharmonic equation $\Delta^{2} u(x)=f(x)$ for $n>4$ or $n=3$ can be represented as

$$
\begin{aligned}
u(x)=\frac{1}{\omega_{n}} \int_{\partial S} \varphi_{0}(\xi) \frac{\partial}{\partial \nu} \Delta_{\xi} G_{2}(x, \xi) d s_{\xi}- & \\
& -\frac{1}{\omega_{n}} \int_{\partial S} \varphi_{1}(\xi) \Delta_{\xi} G_{2}(x, \xi) d s_{\xi}+\frac{1}{\omega_{n}} \int_{S} G_{2}(x, \xi) f(\xi) d \xi
\end{aligned}
$$

where $G_{2}(x, \xi)$ is the Green's function of the Dirichlet problem for the biharmonic equation in the unit ball [3], and $\omega_{n}$ is the area of the unit sphere $\partial S$.

Further, in the paper [28] using the Green's function $G_{2}(x, \xi)$ from [3], the first two surface integrals in the formula (1) are calculated. It turned out that the sum of the values of these integrals can be written in the form

$$
u(x)=u_{0}(x)+\frac{1-|x|^{2}}{2} \Lambda u_{0}(x)-\frac{1-|x|^{2}}{2} u_{1}(x)
$$

where the harmonic functions $u_{0}(x)$ and $u_{1}(x)$ are solutions to the Dirichlet boundary value problems with boundary values $\varphi_{0}(s)$ and $\varphi_{1}(s)$ on $\partial S$, and the differential operator $\Lambda$ has the form $\Lambda u=\sum_{k=1}^{n} x_{k} u_{x_{k}}$. Therefore, the formula (2) can be considered as Green's formula for the Dirichlet problem for the homogeneous biharmonic equation. Since the functions $u_{0}(x)$ and $u_{1}(x)$ can be represented in the integral form through the well-known Green's function $G(x, \xi)$ of the Dirichlet problem for the Poisson equation (see, for example, [29]), then (2) can again be written in integral form. Note that in the papers [15,30], representations of solutions of the Dirichlet and Neumann boundary value problems for biharmonic and polyharmonic equations are also investigated.

Since representation (2) does not formally use the Green's function $G_{2}(x, \xi)$, the idea arose to obtain a formula similar to (2), but without invoking Green's function, to represent the solution of the Dirichlet boundary value problem for the homogeneous $m$-harmonic equation in the unit ball

$$
\begin{gathered}
\Delta^{m} u(x)=0, \quad x \in S, \\
\left.u\right|_{\partial S}=\varphi_{0}(s),\left.\frac{\partial u}{\partial v}\right|_{\partial S}=\varphi_{1}(s), \ldots,\left.\frac{\partial^{m-1} u}{\partial \nu^{m-1}}\right|_{\partial S}=\varphi_{m-1}(s), s \in \partial S,
\end{gathered}
$$

where $v$ is the outward normal to the unit sphere $\partial S$. The implementation of this idea is the aim of the present work. Based on the auxiliary Lemmas 2-4 and Theorem 2, the main result is obtained in Theorem 4. In addition, in Theorem 6, using Lemma 5 and Theorem 4, a solution to the following Neumann boundary value problem

$$
\begin{gathered}
\Delta^{m} u(x)=0, \quad x \in S, \\
\left.\frac{\partial u}{\partial v}\right|_{\partial S}=\psi_{0}(s), \ldots,\left.\frac{\partial^{m-1} u}{\partial v^{m-1}}\right|_{\partial S}=\psi_{m-1}(s), s \in \partial S .
\end{gathered}
$$

is constructed. The required smoothness of the functions $\varphi_{k}(s)$, and $\psi_{k}(s), k=0, \ldots, m-1$ are given below. 


\section{Auxiliary Statements}

Similarly to the representation of the solution to the Dirichlet problem in the biharmonic case (2), we take an $m$-harmonic function in $S$ satisfying the boundary value conditions (4) in the form

$$
u(x)=\sum_{k=0}^{m-1} p_{k}(x)\left(|x|^{2}-1\right)^{k},
$$

where $p_{k}(x) \in C^{m-1}(\bar{S}), k=0, \ldots, m-1$ is a set of harmonic functions in $S$. The above required smoothness of the functions $p_{k}(x)$ is necessary, since the function $u(x)$ must satisfy the boundary conditions (4).

Let $P_{k}(t)=\sum_{s=0}^{k} c_{s} t^{s}$ be some polynomial. We define the "factorial polynomial" corresponding to the polynomial $P_{k}(t)$ by the equality $P_{[k]}(t)=\sum_{s=0}^{k} c_{s} t^{[s]}$, where the factorial monomial $t^{[k]}$ is defined by the equality $t^{[k]}=t(t-1) \cdots(t-k+1)$ [31]. The operator $\Lambda u=\sum_{k=1}^{n} x_{k} u_{x_{k}}$ has an important boundary property

$$
\left.\frac{\partial^{k} u}{\partial v^{k}}\right|_{\partial S}=\left.\Lambda^{[k]} u\right|_{\partial S},
$$

and the function $\Lambda p(x)$ is harmonic if the function $p(x)$ is harmonic.

To apply the operator $\Lambda$ to a product of functions, the following lemma is needed.

Lemma 1 ([13]). Let $p, q \in C^{k}(S)$, then the operator $\Lambda$ satisfies the equality

$$
\Lambda^{[k]}(p q)=\sum_{i=0}^{k}\left(\begin{array}{c}
k \\
i
\end{array}\right) \Lambda^{[i]} p \Lambda^{[k-i]} q .
$$

Let us prove the following main relation.

Theorem 2. Let, for the given harmonic in $S$ functions $q_{i}(x) \in C^{k_{i}}(\bar{S}), i=0, \ldots, m-1$, where $k_{i} \geq 0$, there exist harmonic functions $p_{i}(x) \in C^{m-1}(\bar{S}), i=0, \ldots, m-1$, such that the equalities hold:

$$
\begin{gathered}
r_{s}(x)=\sum_{k=s}^{m-1}(-1)^{k-s}\left(\begin{array}{l}
k \\
s
\end{array}\right) p_{k}(x), \quad s_{j}(x)=\sum_{s=0}^{m-1}(2 s)^{[j]} r_{s}(x), \\
q_{i}(x)=\sum_{j=0}^{i}\left(\begin{array}{l}
i \\
j
\end{array}\right) \Lambda^{[i-j]} s_{j}(x),
\end{gathered}
$$

where $s, j, i=0, \ldots, m-1$. Then, for such $p_{0}(x), \ldots, p_{m-1}(x)$, the m-harmonic function $u(x)$, determined in (7), satisfies the Dirichlet boundary value conditions

$$
\left.\frac{\partial^{i} u}{\partial v^{i}}\right|_{\partial S}=\left.q_{i}(x)\right|_{\partial S}, \quad i=0, \ldots, m-1
$$

Proof. It is easy to see that due to (9), we have

$$
\begin{aligned}
\Lambda^{[i]} \mathcal{u}(x)=\sum_{k=0}^{m-1} \Lambda^{[i]} & \left(p_{k}(x)\left(|x|^{2}-1\right)^{k}\right)=\sum_{k=0}^{m-1} \sum_{j=0}^{i}\left(\begin{array}{l}
i \\
j
\end{array}\right) \Lambda^{[i-j]} p_{k}(x) \Lambda^{[j]}\left(|x|^{2}-1\right)^{k} \\
& =\sum_{k=0}^{m-1} \sum_{j=0}^{i}\left(\begin{array}{c}
i \\
j
\end{array}\right) \Lambda^{[i-j]} p_{k}(x) \sum_{s=0}^{k}(-1)^{k-s}\left(\begin{array}{l}
k \\
s
\end{array}\right)(2 s) \cdots(2 s-j+1)|x|^{2 s},
\end{aligned}
$$


where $i=0, \ldots, m-1$, and therefore, in accordance with the formulas (8) and (10) and the required smoothness of functions $p_{i}(x)$ and $q_{i}(x)$, we obtain

$$
\begin{aligned}
\left.\frac{\partial^{i} u}{\partial v^{i}}\right|_{\partial S}=\Lambda^{[i]} u(x)_{\mid \partial S}=\sum_{j=0}^{i}\left(\begin{array}{c}
i \\
j
\end{array}\right) \Lambda^{[i-j]} \sum_{k=0}^{m-1} \sum_{s=0}^{k}(-1)^{k-s}\left(\begin{array}{l}
k \\
s
\end{array}\right)(2 s)^{[j]} p_{k}(x)_{\mid \partial S} \\
=\sum_{j=0}^{i}\left(\begin{array}{c}
i \\
j
\end{array}\right) \Lambda^{[i-j]} \sum_{s=0}^{m-1}(2 s)^{[j]} \sum_{k=s}^{m-1}(-1)^{k-s}\left(\begin{array}{l}
k \\
s
\end{array}\right) p_{k}(x)_{\mid \partial S} \\
=\sum_{j=0}^{i}\left(\begin{array}{c}
i \\
j
\end{array}\right) \Lambda^{[i-j]} \sum_{s=0}^{m-1}(2 s)^{[j]} r_{S}(x)(x)_{\mid \partial S}=\sum_{j=0}^{i}\left(\begin{array}{l}
i \\
j
\end{array}\right) \Lambda^{[i-j]} s_{j}(x)_{\mid \partial S}=q_{i}(x)_{\mid \partial S,}
\end{aligned}
$$

where $i=0, \ldots, m-1$. The theorem is proved.

Theorem 2 implies that if by the given harmonic in $S$ functions $\left\{q_{i}(x) \in C^{k_{i}}(\bar{S})\right.$, $i=0, \ldots, m-1\}$ we are able to find functions $\left\{s_{j}(x), j=0, \ldots, m-1\right\}$, and then $\left\{r_{s}(x)\right.$, $s=0, \ldots, m-1\}$, and finally, $\left\{p_{k}(x) \in C^{m-1}(\bar{S}), k=0, \ldots, m-1\right\}$ such that the equalities (10) hold, then the $m$-harmonic function $u(x)$ satisfies the boundary conditions (11). Therefore, we need to reverse the transformations (10). This requires auxiliary constructions.

Let a polynomial $P(\lambda)$ be written in factorial monomials

$$
P(\lambda)=\sum_{k=0}^{m} p_{k} \lambda^{[k]}
$$

Consider the following operation of "factorial" differentiation of polynomials

$$
P^{(0)}(\lambda)=P(\lambda), \quad P^{(k)}(\lambda) \equiv\left(P^{(k-1)}(\lambda)\right)^{(1)}=P^{(k-1)}(\lambda+1)-P^{(k-1)}(\lambda) .
$$

It is easy to see that this differentiation reduces the degree of a polynomial by 1 and

$$
\left(\lambda^{[k]}\right)^{(1)}=(\lambda+1)^{[k]}-\lambda^{[k]}=\lambda^{[k-1]}(\lambda+1-(\lambda-k+1))=k \lambda^{[k-1]},
$$

which means that

$$
\left(\lambda^{[k]}\right)^{(j)}=\left\{\begin{array}{ll}
k^{[j]} \lambda^{[k-j],} & k \geq j \\
0, & k<j
\end{array} .\right.
$$

Therefore, (12) implies

$$
P^{(k)}(0)=\left.\sum_{i=k}^{m} p_{i} i^{[k]} \lambda^{[i-k]}\right|_{\lambda=0}=p_{k} k !,
$$

and hence, we have the equality

$$
P(\lambda)=\sum_{k=0}^{m} \frac{P^{(k)}(0)}{k !} \lambda^{[k]} .
$$

Moreover, the definition (13) of the derivative implies the following equality

$$
P^{(k)}(\lambda)=\sum_{i=0}^{k}(-1)^{k-i}\left(\begin{array}{l}
k \\
i
\end{array}\right) P(\lambda+i),
$$

and therefore, coefficients of the polynomial $P(\lambda)$ from (12) can be calculated by the formula

$$
p_{k}=\frac{P^{(k)}(0)}{k !}=\frac{1}{k !} \sum_{i=0}^{k}(-1)^{k-i}\left(\begin{array}{l}
k \\
i
\end{array}\right) P(i) .
$$


The equality (15) can be easily proved by the induction. For $k=1$, it coincides with the definition of the derivative. If it is true for the $(k-1)$ th derivative, then

$$
\begin{aligned}
P^{(k)}(\lambda)= & P^{(k-1)}(\lambda+1)-P^{(k-1)}(\lambda) \\
& =\sum_{i=0}^{k-1}(-1)^{k-1-i}\left(\begin{array}{c}
k-1 \\
i
\end{array}\right) P(\lambda+1+i)+\sum_{i=0}^{k-1}(-1)^{k-i}\left(\begin{array}{c}
k-1 \\
i
\end{array}\right) P(\lambda+i) \\
& =P(\lambda+k)+\sum_{i=1}^{k-1}(-1)^{k-i}\left(\left(\begin{array}{c}
k-1 \\
i-1
\end{array}\right)+\left(\begin{array}{c}
k-1 \\
i
\end{array}\right)\right) P(\lambda+i)+P(\lambda) \\
& =\sum_{i=0}^{k}(-1)^{k-i}\left(\begin{array}{c}
k \\
i
\end{array}\right) P(\lambda+i)
\end{aligned}
$$

and hence the induction step is proved.

Now let us look at the last transformation from (10).

Lemma 2. The last transformation from (10) is invertible in the form

$$
s_{i}(x)=\sum_{j=0}^{i}\left(\begin{array}{l}
i \\
j
\end{array}\right)(-\Lambda)^{[i-j]} q_{j}(x), \quad i=0, \ldots, m-1
$$

Proof. Let the operator matrix $B(\Lambda)$ be of the form $B(\Lambda)=\left\{\left(\begin{array}{l}i \\ j\end{array}\right) \Lambda^{[i-j]}\right\}_{i, j=\overline{0, m-1}}$, where $\left(\begin{array}{l}i \\ j\end{array}\right)=0$ if $i<j$. We prove that $(B(\Lambda))^{-1}=A(\Lambda) \equiv\left\{\left(\begin{array}{l}i \\ j\end{array}\right)(-\Lambda)^{[i-j]}\right\}_{i, j=\overline{0, m-1}}$. Calculate the product $B(\Lambda) A(\Lambda)=\left(c_{i, j}(\Lambda)\right)_{i, j=\overline{0, m-1}}$, where

$$
c_{i, j}(\Lambda)=\sum_{k=0}^{m-1}\left(\begin{array}{l}
i \\
k
\end{array}\right) \Lambda^{[i-k]}\left(\begin{array}{l}
k \\
j
\end{array}\right)(-\Lambda)^{[k-j]} .
$$

Let $i>j$. Since $\left(\begin{array}{l}i \\ k\end{array}\right)\left(\begin{array}{l}k \\ j\end{array}\right)=\left(\begin{array}{l}i \\ j\end{array}\right)\left(\begin{array}{l}i-j \\ k-j\end{array}\right)$, then

$$
c_{i, j}(\Lambda)=\left(\begin{array}{l}
i \\
j
\end{array}\right) \sum_{k=j}^{i}\left(\begin{array}{c}
i-j \\
k-j
\end{array}\right) \Lambda^{[i-k]}(-\Lambda)^{[k-j]}=\left(\begin{array}{l}
i \\
j
\end{array}\right) \sum_{k=0}^{i-j}\left(\begin{array}{c}
i-j \\
k
\end{array}\right) \Lambda^{[i-j-k]}(-\Lambda)^{[k]}
$$

By virtue of the binomial theorem, we have

$$
0=(\Lambda+(-\Lambda))^{[m]}=\sum_{k=0}^{m}\left(\begin{array}{c}
m \\
k
\end{array}\right) \Lambda^{[m-k]}(-\Lambda)^{[k]}
$$

where $m \in \mathbb{N}$. Hence, it follows that in the case $i>j$, we have $c_{i, j}=0$. For $i=j$ from (16), we find

$$
c_{i, i}=\left(\begin{array}{l}
i \\
i
\end{array}\right) \sum_{k=0}^{0}\left(\begin{array}{l}
0 \\
k
\end{array}\right) \Lambda^{[-k]}(-\Lambda)^{[k]}=1 .
$$

If $i<j$, then since $\left(\begin{array}{l}i \\ k\end{array}\right)\left(\begin{array}{l}k \\ j\end{array}\right)=0$, it follows that $c_{i, j}=0$. Therefore, $B(\Lambda) A(\Lambda)=I$, and hence, $(B(\Lambda))^{-1}=A(\Lambda)$. Thus, if $Q(x)=\left(q_{0}(x), \ldots, q_{m-1}(x)\right)^{T}$, and $S(x)=\left(s_{0}(x), \ldots, s_{m-1}(x)\right)^{T}$, then $Q(x)=B(\Lambda) S(x) \Rightarrow S(x)=A(\Lambda) Q(x)$. The lemma is proved.

Consider the following polynomials in $\lambda$

$$
h_{m}(\lambda)=\prod_{k=0}^{m-1}(\lambda-2 k), \quad h_{m, i}(\lambda)=\frac{h_{m}(\lambda)}{(\lambda-2 i) h_{m}^{\prime}(2 i)}, i=0, \ldots, m-1,
$$


where

$$
h_{m}^{\prime}(2 i)=\prod_{k=0, k \neq i}^{m-1}(2 i-2 k)=(-1)^{m-i-1}(2 i) ! !(2 m-2 i-2) ! ! \neq 0 .
$$

It is clear that $\operatorname{deg} h_{m, i}(\lambda)=m-1$. Using the polynomials $h_{m, i}(\lambda)$, in accordance with the formula (12), we can define the coefficients $h_{m, i}^{(j)}$ by the following equalities

$$
h_{m, i}(\lambda)=\sum_{j=0}^{m-1} h_{m, i}^{(j)} \lambda^{[j]}, \quad i=0, \ldots, m-1
$$

By virtue of (14), we have $h_{m, i}^{(j)}=h_{m, i}^{(j)}(0) / j$ !.

Lemma 3. The second transformation from (10) can be reversed in the form

$$
r_{j}(x)=\sum_{i=0}^{m-1} h_{m, j}^{(i)} s_{i}(x), \quad j=0, \ldots, m-1 .
$$

Proof. Denote

$$
C=\left((2 j)^{[i]}\right)_{i, j=\overline{0, m-1}}=\left(\begin{array}{ccccc}
1 & 1 & 1 & \ldots & 1 \\
0 & 2^{[1]} & 4^{[1]} & \ldots & (2 m-2)^{[1]} \\
0 & 2^{[m-1]} & 4^{[m-1]} & \ldots & (2 m-2)^{[m-1]}
\end{array}\right),
$$

and prove that

$$
C^{-1}=\mathbb{H}_{m} \equiv\left(h_{m, i}^{(j)}\right)_{i, j=\overline{0, m-1}} .
$$

It is easy to see that for $i=1, \ldots, m-1$

$$
\left(0,2^{[i]}, \ldots,(2 m-2)^{[i]}\right) \cdot \mathbb{H}_{m}=\left(\sum_{k=0}^{m-1}(2 k)^{[i]} h_{m, k}^{(0)}, \ldots, \sum_{k=0}^{m-1}(2 k)^{[i]} h_{m, k}^{(m-1)}\right)
$$

Consider the following polynomials of degree $m-1$

$$
g_{i}(\lambda)=\sum_{k=0}^{m-1}(2 k)^{[i]} h_{m, k}(\lambda)=\sum_{k=0}^{m-1} \frac{(2 k)^{[i]}}{h_{m}^{\prime}(2 k)} \frac{h_{m}(\lambda)}{(\lambda-2 k)} .
$$

Using the notation of the formula (18), we write

$$
g_{i}(\lambda)=\sum_{k=0}^{m-1}(2 k)^{[i]} \sum_{j=0}^{m-1} h_{m, k}^{(j)} \lambda^{[j]}=\sum_{j=0}^{m-1} \lambda^{[j]} \sum_{k=0}^{m-1}(2 k)^{[i]} h_{m, k}^{(j)} \equiv \sum_{j=0}^{m-1} g_{i}^{(j)} \lambda^{[j]},
$$

and therefore, (20) can be written as

$$
\left(0,2^{[i]}, \ldots,(2 m-2)^{[i]}\right) \cdot \mathbb{H}_{m}=\left(g_{i}^{(0)}, g_{i}^{(1)}, \ldots, g_{i}^{(m-1)}\right) .
$$

It is not hard to see that for $j=0, \ldots, m-1$, the equalities

$$
g_{i}(2 j)=\sum_{k=0}^{m-1} \frac{(2 k)^{[i]}}{h_{m}^{\prime}(2 k)} \frac{h_{m}(2 j)}{(2 j-2 k)}=\frac{(2 j)^{[i]}}{h_{m}^{\prime}(2 j)} h_{m}^{\prime}(2 j)=(2 j)^{[i]}
$$

hold. Since $g_{i}(\lambda)$ is a polynomial of degree $m-1$ and the equalities $\left(g_{i}(\lambda)-\lambda^{[i]}\right)_{\mid \lambda=2 j}=0$ are true at $m$ points $\lambda=2 j$, where $j=0, \ldots, m-1$, then $g_{i}(\lambda) \equiv \lambda^{[i]}$. Therefore, from (21) it follows $\left(0,2^{[i]}, \ldots,(2 m-2)^{[i]}\right) \cdot \mathbb{H}_{m}=e_{i}$ which means $C \mathbb{H}_{m}=I$. Thus, if 
$S(x)=\left(s_{0}(x), \ldots, s_{m-1}(x)\right)^{T}$ and $R(x)=\left(r_{0}(x), \ldots, r_{m-1}(x)\right)^{T}$, then $S(x)=C R(x) \Rightarrow$ $R(x)=\mathbb{H}_{m} S(x)$. The lemma is proved.

Remark 1. In the paper ([32] [Theorem 4]), it is established that the following connection between the $k$ th $(0 \leq k \leq m-1)$ row of the matrix $\mathbb{H}_{m}$ and the coefficients in the representation of the value $\Delta^{k} u(0)(u(x)$ is m-harmonic in $S$ function) through the values of its normal derivatives in the form

$$
\Delta^{k} u(0)=\frac{(2,2)_{k}(n, 2)_{k}}{\omega_{n}} \int_{\partial S} \sum_{j=0}^{m-1} h_{m, k}^{(j)} \frac{\partial^{j} u}{\partial v^{j}} d s_{\tilde{\zeta}}, \quad k=0, \ldots, m-1,
$$

where $(a, b)_{k}=a(a+b) \ldots(a+(k-1) b)$ is the generalized Pochhammer symbol with convention $(a, b)_{0}=1$.

Remark 2. The last row of the matrix $\mathbb{H}_{m}$ gives the solvability condition of the Neumann boundary value problem for the $(m-1)$-harmonic equation with boundary conditions: $\left.\frac{\partial^{j} u}{\partial v^{j}}\right|_{\partial S}=\varphi_{j}$, $j=1, \ldots, m-1$ (see [33] [Theorem 10]) in the form

$$
\int_{\partial S} \sum_{j=1}^{m-1} h_{m, m-1}^{(j)} \varphi_{j} d s_{\xi}=0
$$

Example 1. Let us find the matrix $\mathbb{H}_{4}$. Since $h_{4}(\lambda)=\prod_{k=0}^{3}(\lambda-2 k)=\lambda(\lambda-2)(\lambda-4)(\lambda-6)$, then

$$
h_{4}^{\prime}(2 i)=(-1)^{3-i} \frac{(6) ! !}{C_{3}^{i}}
$$

Therefore, according to (17),

$$
h_{4, i}(\lambda)=\frac{h_{4}(\lambda)}{(\lambda-2 i) h_{4}^{\prime}(2 i)}=(-1)^{3-i} \frac{C_{3}^{i}}{(6) ! !} \prod_{k=0, k \neq i}^{3}(\lambda-2 k) .
$$

From here, we can find $h_{4,0}(\lambda)=-\frac{1}{6 ! !}(\lambda-2)(\lambda-4)(\lambda-6)$. If we use (15)

$$
h_{4,0}^{(k)}(0)=\sum_{i=0}^{k}(-1)^{k-i}\left(\begin{array}{c}
k \\
i
\end{array}\right) h_{4,0}(i) .
$$

Then, we obtain

$$
\begin{gathered}
h_{4,0}^{(0)}(0)=-\frac{1}{6 ! !}(-2)(-4)(-6)=1, \\
h_{4,0}^{(1)}(0)=-\frac{1}{6 ! !}((-1)(-3)(-5)-(-2)(-4)(-6))=-\frac{33}{6 ! !}, \\
h_{4,0}^{(2)}(0)=-\frac{1}{6 ! !}(0-2 \cdot(-1)(-3)(-5)+(-2)(-4)(-6))=\frac{18}{6 ! !}, \\
h_{4,0}^{(3)}(0)=-\frac{1}{6 ! !}(1(-1)(-3)-3 \cdot 0+3 \cdot(-1)(-3)(-5)-(-2)(-4)(-6))=-\frac{6}{6 ! !},
\end{gathered}
$$

whence by virtue of (14)

$$
h_{4,0}(\lambda)=\sum_{k=0}^{3} \frac{h_{4,0}^{(k)}(0)}{k !} \lambda^{[k]}=1-\frac{33}{48} \lambda^{[1]}+\frac{9}{48} \lambda^{[2]}-\frac{1}{48} \lambda^{[3]},
$$


and therefore, the first row of the matrix $\mathbb{H}_{4}$ has the form $\left(1,-\frac{11}{16}, \frac{3}{16},-\frac{1}{48}\right)$. Similarly, we can find $h_{4,1}(\lambda)=\frac{3}{6 ! !} \lambda(\lambda-4)(\lambda-6), h_{4,2}(\lambda)=-\frac{3}{6 ! !} \lambda(\lambda-2)(\lambda-6), h_{4,3}(\lambda)=\frac{1}{6 ! !} \lambda(\lambda-2)(\lambda-4)$, whence we get

$$
\mathbb{H}_{4}=\left(\begin{array}{cccc}
1 & -\frac{11}{16} & \frac{3}{16} & -\frac{1}{48} \\
0 & \frac{15}{16} & -\frac{7}{16} & \frac{1}{16} \\
0 & -\frac{5}{16} & \frac{5}{16} & -\frac{1}{16} \\
0 & \frac{1}{16} & -\frac{1}{16} & \frac{1}{48}
\end{array}\right) .
$$

According to (22), for the four-harmonic function $u(x)$, the first row of the matrix $\mathbb{H}_{4}$ gives the equality

$$
u(0)=\frac{1}{\omega_{n}} \int_{\partial S}\left(u-\frac{11}{16} \frac{\partial u}{\partial v}+\frac{3}{16} \frac{\partial^{2} u}{\partial v^{2}}-\frac{1}{48} \frac{\partial^{3} u}{\partial v^{3}}\right) d s_{\xi} .
$$

Moreover, according to (23), the fourth row of the matrix $\mathbb{H}_{4}$ gives the solvability condition of the Neumann boundary value problem for the three-harmonic equation

$$
\int_{\partial S}\left(3 \varphi_{1}-3 \varphi_{2}+\varphi_{3}\right) d s_{\xi}=0 .
$$

Lemma 4. The first transformation from (10) can be reversed in the form

$$
p_{k}(x)=\sum_{j=k}^{m-1}\left(\begin{array}{l}
j \\
k
\end{array}\right) r_{j}(x), \quad k=0, \ldots, m-1 .
$$

Proof. By Lemma 11 from [13], we can write

$$
C^{-1} \equiv\left\{(-1)^{j-i}\left(\begin{array}{l}
j \\
i
\end{array}\right)\right\}_{i, j=\overline{0, m-1}}^{-1}=\left\{\left(\begin{array}{l}
j \\
i
\end{array}\right)\right\}_{i, j=\overline{0, m-1}} .
$$

Thus, if $P(x)=\left(p_{0}(x), \ldots, p_{m-1}(x)\right)^{T}$ and $R(x)=\left(r_{0}(x), \ldots, r_{m-1}(x)\right)^{T}$, then $R(x)=C P(x) \Rightarrow P(x)=\mathbb{C}^{-1} R(x)$. This proves the lemma.

\section{Inverting the Main Relation}

The following statement follows from Lemmas $2-4$ and Theorem 2.

Theorem 3. Let $q_{i}(x) \in C^{2 m-2-i}(\bar{S}), i=0, \ldots, m-1$ be some system of a harmonic in $S$ functions and a harmonic in $S$ functions $p_{s}(x)$, defined by the equalities

$$
p_{s}(x)=\sum_{j=0}^{s} \frac{1}{j !} H_{s}^{(j)}(-\Lambda) q_{j}(x), \quad s=0, \ldots, m-1,
$$

where

$$
H_{s}(\lambda)=\frac{1}{(2 s) ! !} \lambda(\lambda-2) \cdots(\lambda-2 s+2), \quad s \in \mathbb{N},
$$

with convention $H_{0}(\lambda)=1$, and the $j$ th-order derivative $H_{s}^{(j)}(\lambda)$ of the polynomial $H_{s}(\lambda)$ is taken in the sense of definition (13). Then the m-harmonic function

$$
u(x)=\sum_{k=0}^{m-1} p_{k}(x)\left(|x|^{2}-1\right)^{k}
$$

satisfies the boundary conditions of the Dirichlet boundary value problem (11). 
Proof. Let us use Theorem 2 and consider the equalities (10). If we consistently apply Lemmas 2-4, then the equalities (10) can be inverted in the form

$$
p_{s}(x)=\sum_{k=s}^{m-1}\left(\begin{array}{l}
k \\
s
\end{array}\right) \sum_{i=0}^{m-1} h_{m, k}^{(i)} \sum_{j=0}^{i}\left(\begin{array}{l}
i \\
j
\end{array}\right)(-\Lambda)^{[i-j]} q_{j}(x), \quad s=0, \ldots, m-1,
$$

where the numbers $h_{m, k}^{(i)}$ are the elements of the matrix $\mathbb{H}_{m}$ from (19). Therefore, by Theorem 2, for such polynomials $p_{s}(x)$, the function $u(x)$ from (28) satisfies the Dirichlet boundary conditions (11).

Simplify the equalities (29). It is not hard to see that

$$
p_{s}(x)=\sum_{k=s}^{m-1}\left(\begin{array}{l}
k \\
s
\end{array}\right) \sum_{i=0}^{m-1} h_{m, k}^{(i)} \psi(i)=\sum_{i=0}^{m-1} \psi(i) \sum_{k=s}^{m-1}\left(\begin{array}{l}
k \\
s
\end{array}\right) h_{m, k^{\prime}}^{(i)}
$$

where $\psi(i)$ denotes the internal sum over $j$ in (29). Consider the following polynomial

$$
H_{s}(\lambda)=\sum_{k=s}^{m-1}\left(\begin{array}{l}
k \\
s
\end{array}\right) h_{m, k}(\lambda)
$$

where the polynomials $h_{m, k}(\lambda)$ are defined in (17). Using (24), the polynomial $h_{m, k}(\lambda)$ can be written in the form

$$
\begin{aligned}
h_{m, k}(\lambda)=\frac{h_{m}(\lambda)}{(\lambda-2 k) h^{\prime}(2 k)}=\frac{(-1)^{m-k-1}}{(2 m-2) ! !}\left(\begin{array}{c}
m-1 \\
k
\end{array}\right) & \frac{h_{m}(\lambda)}{(\lambda-2 k)} \\
& =\frac{(-1)^{m-k-1}}{(2 k) ! !(2 m-2-2 k) ! !} \frac{h_{m}(\lambda)}{(\lambda-2 k)} .
\end{aligned}
$$

Here, as in (17), it is denoted that $h_{m}(\lambda)=\lambda(\lambda-2) \cdots(\lambda-2 m+2)$. Therefore, we have

$$
\begin{gathered}
H_{s}(\lambda)=\sum_{k=s}^{m-1}\left(\begin{array}{l}
k \\
s
\end{array}\right) h_{m, k}(\lambda)=\frac{h_{m}(\lambda)}{(2 s) ! !} \sum_{k=s}^{m-1} \frac{(-1)^{m-k-1}}{(2 k-2 s) ! !(2 m-2-2 k) ! !} \frac{1}{\lambda-2 k} \\
=\frac{h_{m}(\lambda)}{(2 s) ! !(2 m-2-2 s) ! !} \sum_{k=s}^{m-1}\left(\begin{array}{c}
m-1-s \\
k-s
\end{array}\right) \frac{(-1)^{m-k-1}}{\lambda-2 k} \\
=\frac{h_{m}(\lambda)}{(2 s) ! !(2 m-2-2 s) ! !} \sum_{k=0}^{m-1-s}\left(\begin{array}{c}
m-1-s \\
k
\end{array}\right) \frac{(-1)^{m-k-s-1}}{\lambda-2 k-2 s} .
\end{gathered}
$$

Next, we use the simple equality

$$
\sum_{k=0}^{m}\left(\begin{array}{l}
m \\
k
\end{array}\right) \frac{(-1)^{m-k}}{\mu-2 k}=\frac{(2 m) ! !}{\mu(\mu-2) \cdots(\mu-2 m)}
$$


which is obvious for $m=0$ and can be easily proved by the induction on $m>0$ :

$$
\begin{aligned}
& \frac{(2 m) ! !}{\mu(\mu-2) \cdots(\mu-2 m)}=\frac{(2 m-2) ! !}{(\mu-2) \cdots(\mu-2 m+2)}\left(\frac{1}{\mu-2 m}-\frac{1}{\mu}\right) \\
&=\sum_{k=0}^{m-1}\left(\begin{array}{c}
m-1 \\
k
\end{array}\right) \frac{(-1)^{m-1-k}}{\mu-2 k-2}-\sum_{k=0}^{m-1}\left(\begin{array}{c}
m-1 \\
k
\end{array}\right) \frac{(-1)^{m-1-k}}{\mu-2 k} \\
&=\sum_{k=1}^{m}\left(\begin{array}{c}
m-1 \\
k-1
\end{array}\right) \frac{(-1)^{m-k}}{\mu-2 k}+\sum_{k=0}^{m}\left(\begin{array}{c}
m-1 \\
k
\end{array}\right) \frac{(-1)^{m-k}}{\mu-2 k} \\
&=\sum_{k=0}^{m}\left(\left(\begin{array}{c}
m-1 \\
k-1
\end{array}\right)+\left(\begin{array}{c}
m-1 \\
k
\end{array}\right)\right) \frac{(-1)^{m-k}}{\mu-2 k}=\sum_{k=0}^{m}\left(\begin{array}{c}
m \\
k
\end{array}\right) \frac{(-1)^{m-k}}{\mu-2 k} .
\end{aligned}
$$

Thus, from (31) for $m^{\prime}=m-1-s$ and $\mu=\lambda-2 s$, we get

$$
H_{s}(\lambda)=\sum_{k=s}^{m-1}\left(\begin{array}{l}
k \\
s
\end{array}\right) h_{m, k}(\lambda)=\frac{h_{m}(\lambda)}{(2 s) ! !(\lambda-2 s) \cdots(\lambda-2 m+2)} .
$$

Hence, for $s=0$, we find $H_{0}(\lambda)=1$. If $s=1, \ldots, m-1$, then using the value of $h_{m, k}(\lambda)$ from (17) we obtain $H_{s}(\lambda)=\frac{1}{(2 s) ! !} \lambda(\lambda-2) \cdots(\lambda-2 s+2)$, which is the same as the polynomial defined in (27). Further, we expand the polynomial $H_{S}(\lambda)$ in monomial powers $\lambda^{[i]}$ as in (14)

$$
H_{s}(\lambda)=\sum_{i=0}^{s} \frac{H_{s}^{(i)}(0)}{i !} \lambda^{[i]} .
$$

By the definition of $H_{s}(\lambda)$ and using (18), we get

$$
H_{s}(\lambda)=\sum_{k=s}^{m-1}\left(\begin{array}{l}
k \\
s
\end{array}\right) h_{m, k}(\lambda)=\sum_{k=s}^{m-1}\left(\begin{array}{l}
k \\
s
\end{array}\right) \sum_{i=0}^{m-1} h_{m, k}^{(i)} \lambda^{[i]}=\sum_{i=0}^{m-1} \lambda^{[i]} \sum_{k=s}^{m-1}\left(\begin{array}{l}
k \\
s
\end{array}\right) h_{m, k^{\prime}}^{(i)}
$$

whence it follows that

$$
\sum_{k=s}^{m-1}\left(\begin{array}{l}
k \\
s
\end{array}\right) h_{m, k}^{(i)}=\frac{H_{s}^{(i)}(0)}{i !}
$$

Substituting the found value of the sum into the equality (30) and using the definition of $\psi(i)$, we get

$$
p_{s}(x)=\sum_{i=0}^{s} \frac{H_{s}^{(i)}(0)}{i !} \sum_{j=0}^{i}\left(\begin{array}{l}
i \\
j
\end{array}\right)(-\Lambda)^{[i-j]} q_{j}(x), \quad s=0, \ldots, m-1 .
$$

Reversing the order of summations and using the expansion of the polynomial $H_{s}^{(j)}(\lambda)$ in the form (14), we have

$$
p_{s}(x)=\sum_{j=0}^{s} \frac{1}{j !}\left(\sum_{i=j}^{s} \frac{\left(H_{s}^{(j)}\right)^{(i-j)}(0)}{(i-j) !}(-\Lambda)^{[i-j]}\right) q_{j}(x)=\sum_{j=0}^{s} \frac{1}{j !} H_{s}^{(j)}(-\Lambda) q_{j}(x),
$$

where $s=0, \ldots, m-1$, which is the same as (26).

Let us check the smoothness of the functions $p_{s}(x)$. In Theorem 2, it is required that $p_{0}(x), \ldots, p_{m-1}(x) \in C^{m-1}(\bar{S})$. Since $\operatorname{deg} H_{s}^{(j)}(\lambda)=s-j$, then for such smoothness from the formula (26), for example for $s=m-1$, it follows that $q_{j}(x) \in C^{2 m-2-j}(\bar{S})$. For $s<m-1$, the smoothness conditions for $q_{j}(x)$ are weaker than those indicated. Hence, in Theorem 2, we can take $k_{j}=2 m-2-j, j=0, \ldots, m-1$. The theorem is proved. 
Remark 3. The formula (28) is similar to the well-known Almansi formula [34,35], but in (28), the harmonic components are known.

\section{Dirichlet Boundary Value Problem}

The assertions proved above allow us to formulate the main result on the Dirichlet boundary value problem.

Theorem 4. Solution of the Dirichlet boundary value problem for the polyharmonic equation

$$
\begin{gathered}
\Delta^{m} u(x)=f(x), \quad x \in S, \\
\left.u\right|_{\partial S}=\varphi_{0}(x),\left.\quad \frac{\partial u}{\partial v}\right|_{\partial S}=\varphi_{1}(s), \quad \cdots,\left.\quad \frac{\partial^{m-1} u}{\partial v^{m-1}}\right|_{\partial S}=\varphi_{m-1}(s), \quad s \in \partial S,
\end{gathered}
$$

for $f \in C^{1}(\bar{S}), \varphi_{k} \in C^{2 m-2-k+\varepsilon}(\partial S), k=0, \ldots, m-1, \varepsilon>0$ can be written as

$$
u(x)=\sum_{j=0}^{m-1} \frac{1}{j !} K_{m-1}^{(j)}\left(-\Lambda ;|x|^{2}-1\right) q_{j}(x)+\int_{S} G_{m}(x, \xi) f(\xi) d \xi,
$$

where

$$
K_{m-1}\left(\lambda ;|x|^{2}-1\right)=\sum_{k=0}^{m-1}\left(|x|^{2}-1\right)^{k} H_{k}(\lambda)
$$

the polynomials $H_{k}(\lambda)$ are determined from $(27)$, the derivative $K_{m-1}^{(j)}\left(\lambda ;|x|^{2}-1\right)$ with respect to $\lambda$ of order $j$ is taken in the sense of the definition (13), and the harmonic functions $q_{k}(x)$, $k=0, \ldots, m-1$ are solutions of the Dirichlet problems

$$
\Delta q_{k}(x)=0, x \in S ;\left.\quad q_{k}\right|_{\partial S}=\varphi_{k}(s), s \in \partial S,
$$

and $G_{m}(x, \xi)$ is the Green's function of the Dirichlet boundary value problem in the unit ball for the m-harmonic equation.

Proof. Let $q_{i}(x) \in C^{2 m-2-i}(\bar{S}), i=0, \ldots, m-1$ be a system of harmonic functions in $S$ that are solutions to problems (34). Then, by Theorem 3 and using the properties of Green's function $G_{m}(x, \xi)$, the function

$$
u(x)=\sum_{k=0}^{m-1}\left(|x|^{2}-1\right)^{k} p_{k}(x)+\int_{S} G_{m}(x, \xi) f(\xi) d \xi,
$$

where the harmonic functions $p_{k}(x)$ for $k=0, \ldots, m-1$ are found from the equalities

$$
p_{k}(x)=\sum_{j=0}^{k} \frac{1}{j !} H_{k}^{(j)}(-\Lambda) q_{j}(x), \quad k=0, \ldots, m-1,
$$

the polynomial $H_{k}(\lambda)$ has the form $H_{k}(\lambda)=\frac{1}{(2 k) ! !} \lambda(\lambda-2) \cdots(\lambda-2 k+2), k \in \mathbb{N}$, and the $j$ th-order derivative $H_{k}^{(j)}(\lambda)$ is taken in the sense of the definition (13), and satisfies the Dirichlet boundary conditions (4)

$$
\left.\frac{\partial^{i} u}{\partial v^{i}}\right|_{\partial S}=\left.q_{i}(x)\right|_{\partial S}+\left.\int_{S} \frac{\partial^{i} G_{m}(x, \xi)}{\partial v_{x}^{i}} f(\xi) d \xi\right|_{x \in \partial S}=\varphi_{i}(s), s \in \partial S,
$$


for $i=0, \ldots, m-1$. We transform the first term in the resulting solution (35). It is easy to see that using the notation (33), we can write

$$
\begin{aligned}
& \sum_{k=0}^{m-1}\left(|x|^{2}-1\right)^{k} p_{k}(x)=\sum_{k=0}^{m-1}\left(|x|^{2}-1\right)^{k} \sum_{j=0}^{k} \frac{1}{j !} H_{k}^{(j)}(-\Lambda) q_{j}(x) \\
& \quad=\left.\sum_{j=0}^{m-1} \frac{1}{j !}\left(\sum_{k=j}^{m-1}\left(|x|^{2}-1\right)^{k} H_{k}(\mu)\right)_{\mu}^{(j)}\right|_{\mu=-\Lambda} q_{j}(x)=\sum_{j=0}^{m-1} \frac{1}{j !} K_{m-1}^{(j)}\left(-\Lambda ;|x|^{2}-1\right) q_{j}(x),
\end{aligned}
$$

where it is taken into account that the $j$ th-order derivative of $H_{k}(\mu)$ for $k<j$ is equal to zero. Finally, by virtue of paper ([36] [Lemma 2.7]), in order for the harmonic in $S$ functions $q_{i}(x)$ to have the smoothness $q_{i}(x) \in C^{2 m-2-i}(\bar{S})$, it is sufficient to require that $\varphi_{k} \in C^{2 m-2-k+\varepsilon}(\partial S), k=0, \ldots, m-1$ for some $\varepsilon>0$. Therefore, the $m$-harmonic function $u(x)$ from (32) is a solution to the Dirichlet boundary value problem (3) and (4). The theorem is proved.

Example 2. Let us find the solution to the Dirichlet boundary value problem for the three-harmonic equation in the unit ball. In this case $m=3$. First, find the polynomials $H_{0}(\lambda), H_{1}(\lambda)$ and $H_{2}(\lambda)$ by the formula (27). We have

$$
H_{0}(\lambda)=1, \quad H_{1}(\lambda)=\frac{1}{2} \lambda^{[1]}, \quad H_{2}(\lambda)=\frac{1}{4 ! !} \lambda(\lambda-2)=\frac{1}{4 ! !}\left(\lambda^{[2]}-\lambda^{[1]}\right),
$$

which means

$$
K\left(\lambda ;|x|^{2}-1\right)=\sum_{k=0}^{2}\left(|x|^{2}-1\right)^{k} H_{k}(\lambda)=1+\frac{1}{2}\left(|x|^{2}-1\right) \lambda^{[1]}+\frac{1}{4 ! !}\left(|x|^{2}-1\right)^{2}\left(\lambda^{[2]}-\lambda^{[1]}\right) .
$$

If we recall the equality $\left(\lambda^{[k]}\right)^{(m)}=k^{[m]} \lambda^{[k-m]}$, then it is not hard to find

$$
\begin{gathered}
K_{\lambda}^{(1)}\left(\lambda ;|x|^{2}-1\right)=\frac{1}{2}\left(|x|^{2}-1\right)+\frac{1}{4 ! !}\left(|x|^{2}-1\right)^{2}\left(2 \lambda^{[1]}-1\right), \\
K_{\lambda}^{(2)}\left(\lambda ;|x|^{2}-1\right)=\frac{2}{4 ! !}\left(|x|^{2}-1\right)^{2} .
\end{gathered}
$$

Hence, by the formula (32), using ([4] [Theorem 2]), we can write

$$
\begin{aligned}
& u(x)=\sum_{j=0}^{2} \frac{1}{j !} K_{\lambda}^{(j)}\left(-\Lambda ;|x|^{2}-1\right) q_{j}(x)-\frac{1}{\omega_{n}} \int_{S} G_{3}(x, \xi) f(\xi) d \xi \\
&=\left(1-\frac{1}{2}\left(|x|^{2}-1\right) \Lambda+\frac{1}{4 ! !}\left(|x|^{2}-1\right)^{2}\left(\Lambda^{2}+2 \Lambda\right)\right) q_{0}(x) \\
&+\left(\frac{1}{2}\left(|x|^{2}-1\right)-\frac{1}{4 ! !}\left(|x|^{2}-1\right)^{2}(2 \Lambda+1)\right) q_{1}(x)+\frac{1}{4 ! !}\left(|x|^{2}-1\right)^{2} q_{2}(x) \\
&-\frac{1}{\omega_{n}} \int_{S} G_{3}(x, \xi) f(\xi) d \xi
\end{aligned}
$$

where $G_{3}(x, \xi)$ is the Green's function [4] of the Dirichlet boundary value problem for $m=3$.

The possibility of recursive construction of the solution (32) to the Dirichlet boundary value problem (3) and (4) for the homogeneous polyharmonic equation is given in the following theorem.

Theorem 5. Let $u_{m}(x)$ be a solution of the Dirichlet boundary value problem (3) and (4) with $f(x)=0$, written as (32). Then, the $(m+1)$-harmonic function $u_{m+1}(x)$-a solution to the Dirich- 
let boundary value problem (3) and (4) with the same boundary functions $\varphi_{k} \in C^{2 m-2-k+\varepsilon}(\partial S)$, $k=0, \ldots, m-1$ and $\varphi_{m} \in C^{m+\varepsilon}(\partial S)$ can be written as

$$
u_{m+1}(x)=u_{m}(x)+\left(|x|^{2}-1\right)^{m} \sum_{j=0}^{m} \frac{1}{j !} H_{m}^{(j)}(-\Lambda) q_{j}(x),
$$

where the polynomial $H_{m}(\lambda)$ is defined in (27), the derivative $H_{m}^{(j)}(\lambda)$ is taken in the sense of (13), and the harmonic functions $q_{k}(x), k=0, \ldots, m-1$ are solutions of the corresponding Dirichlet boundary value problems, as in Theorem 4.

Proof. By Theorem 4, solutions of the Dirichlet boundary value problems $u_{m+1}(x)$ and $u_{m}(x)$ can be written as (32). Transform the difference of these solutions

$$
\begin{gathered}
u_{m+1}(x)-u_{m}(x)=\sum_{j=0}^{m-1} \frac{1}{j !}\left(K_{m}^{(j)}\left(-\Lambda ;|x|^{2}-1\right)+\frac{1}{m !} K_{m}^{(m)}\left(-\Lambda ;|x|^{2}-1\right) q_{m}(x)\right. \\
\left.-K_{m-1}^{(j)}\left(-\Lambda ;|x|^{2}-1\right)\right) q_{j}(x)=\left(|x|^{2}-1\right)^{m} \sum_{j=0}^{m-1} \frac{1}{j !} H_{m}^{(j)}(-\Lambda) q_{j}(x) \\
\quad+\frac{1}{m !}\left(|x|^{2}-1\right)^{m} H_{m}^{(m)}(-\Lambda) q_{m}(x)=\left(|x|^{2}-1\right)^{m} \sum_{j=0}^{m} \frac{1}{j !} H_{m}^{(j)}(-\Lambda) q_{j}(x) .
\end{gathered}
$$

This equality proves the theorem.

Example 3. Let us use the formula (37) to construct a solution to the Dirichlet boundary value problem for the homogeneous four-harmonic equation using the solution (36) of the Dirichlet boundary value problem for the homogeneous three-harmonic equation obtained in Example 2. To do this, we need to use the "factorial" representation of the polynomial $H_{3}(\lambda)$ given in Example 1. Its coefficients are located in the last row of the matrix $\mathbb{H}_{4}$ from (25):

$$
H_{3}(\lambda)=\frac{1}{6 ! !} \lambda(\lambda-2)(\lambda-4)=\frac{1}{6 ! !}\left(3 \lambda^{[1]}-3 \lambda^{[2]}+\lambda^{[3]}\right) .
$$

Using the equality $\left(\lambda^{[k]}\right)^{(m)}=k^{[m]} \lambda^{[k-m]}$, we get

$$
H_{3}^{(1)}(\lambda)=\frac{1}{6 ! !}\left(3-6 \lambda^{[1]}+3 \lambda^{[2]}\right), H_{3}^{(2)}(\lambda)=\frac{1}{6 ! !}\left(-6+6 \lambda^{[1]}\right), H_{3}^{(3)}(\lambda)=1,
$$

and hence, by (37) for $m=3$, we obtain

$$
\begin{aligned}
u_{4}(x) & -u_{3}(x) \\
& =\frac{\left(|x|^{2}-1\right)^{3}}{6 ! !}\left(-\left(\Lambda^{3}+6 \Lambda^{2}+8 \Lambda\right) q_{0}+\left(3 \Lambda^{2}+9 \Lambda+3\right) q_{1}-(3 \Lambda+3) q_{2}+q_{3}\right) .
\end{aligned}
$$

From here, using (36), we find

$$
\begin{aligned}
& u_{4}(x)= \\
& \begin{aligned}
=\left(1-\frac{\left(|x|^{2}-1\right)}{2} \Lambda\right. & \left.+\frac{\left(|x|^{2}-1\right)^{2}}{4 ! !}\left(\Lambda^{2}+2 \Lambda\right)-\frac{\left(|x|^{2}-1\right)^{3}}{6 ! !}\left(\Lambda^{3}+6 \Lambda^{2}+8 \Lambda\right)\right) q_{0}(x) \\
& +\left(\frac{\left(|x|^{2}-1\right)}{2}-\frac{\left(|x|^{2}-1\right)^{2}}{4 ! !}(2 \Lambda+1)+\frac{\left(|x|^{2}-1\right)^{3}}{6 ! !}\left(3 \Lambda^{2}+9 \Lambda+3\right)\right) q_{1}(x) \\
& +\left(\frac{\left(|x|^{2}-1\right)^{2}}{4 ! !}-\frac{\left(|x|^{2}-1\right)^{3}}{6 ! !}(3 \Lambda+3)\right) q_{2}(x)+\frac{\left(|x|^{2}-1\right)^{3}}{6 ! !} q_{3}(x) .
\end{aligned}
\end{aligned}
$$




\section{Neumann Boundary Value Problem}

Now consider the Neumann boundary value problem (5) and (6).

Theorem 6. Let the harmonic functions $q_{k}(x)$ be solutions of the Dirichlet boundary value problems

$$
\Delta q_{k}(x)=0, x \in S ;\left.\quad q_{k}\right|_{\partial S}=\psi_{k}(s), s \in \partial S,
$$

where $\psi_{k} \in C^{2 m-1-k+\varepsilon}(\partial S), k=0, \ldots, m-1, \varepsilon>0$. Then, for the existence of a solution to the Neumann boundary value problem (5) and (6)

$$
\begin{gathered}
\Delta^{m} u(x)=0, \quad x \in S, \\
\left.\frac{\partial u}{\partial v}\right|_{\partial S}=\psi_{0}(s), \quad \cdots,\left.\quad \frac{\partial^{m-1} u}{\partial v^{m-1}}\right|_{\partial S}=\psi_{m-1}(s), \quad s \in \partial S,
\end{gathered}
$$

it is necessary and sufficient to fulfill the condition

$$
\sum_{j=0}^{m-1} h_{m}^{(j+1)} q_{j}(0)=0,
$$

where $h_{m}^{(j)}$ are coefficients from the representation $h_{m}(\lambda) \equiv \lambda(\lambda-2) \ldots(\lambda-2 m+2)=$ $\sum_{j=0}^{m-1} h_{m}^{(j)} \lambda^{[j]}$. The solution of the Neumann problem can be written as

$$
u(x)=\int_{0}^{1} v(t x) \frac{d t}{t}+C
$$

where $C$ is an arbitrary constant,

$$
v(x)=\sum_{j=0}^{m-1} \frac{1}{j !} K_{m-1}^{(j)}\left(1-\Lambda ;|x|^{2}-1\right) q_{j}(x),
$$

and $K_{m-1}^{(j)}\left(\lambda ;|x|^{2}-1\right)$ is determined in (33).

Proof. Let us make, in the Neumann boundary value problem, (5) and (6) be the change of the variable $v=\Lambda u$. Then, since $\Delta \Lambda u(x)=(\Lambda+2) \Delta u(x)$, whence follows $\Delta^{m} \Lambda u(x)=(\Lambda+2 m) \Delta^{m} u(x)=0$, we get the following boundary value problem for $v(x)$

$$
\Delta^{m} v(x)=0, x \in S,\left.\quad v\right|_{\partial S}=\psi_{0}(s), \ldots,\left.(\Lambda-1)^{[m-1]} v\right|_{\partial S}=\psi_{m-1}(s), s \in \partial S .
$$

By Theorem 4, in which we formally replace the operator $\Lambda$ by the operator $\Lambda-1$, the solution to this problem can be written in the form

$$
v(x)=\sum_{j=0}^{m-1} \frac{1}{j !} K_{m-1}^{(j)}\left(1-\Lambda ;|x|^{2}-1\right) q_{j}(x),
$$

where the harmonic functions $q_{j}(x)$ are solutions to the problems (38). Denote

$$
\begin{gathered}
v_{1}(x)=\sum_{j=0}^{m-1} \frac{1}{j !} K_{m-1}^{(j)}\left(1-\Lambda ;|x|^{2}-1\right)\left(q_{j}(x)-q_{j}(0)\right), \\
v_{0}(x)=\sum_{j=0}^{m-1} \frac{q_{j}(0)}{j !} K_{m-1}^{(j)}\left(1 ;|x|^{2}-1\right) .
\end{gathered}
$$


It is easy to see that since $\Lambda\left(q_{j}(0)\right)=0$ and $K_{m-1}^{(j)}\left(\lambda ;|x|^{2}-1\right)$ is a polynomial in $\lambda$, then the following equalities hold true:

$$
\begin{aligned}
v(x)=\sum_{j=0}^{m-1} \frac{1}{j !} K_{m-1}^{(j)}\left(1-\Lambda ;|x|^{2}-1\right) & \left(q_{j}(x)-q_{j}(0)\right) \\
+ & \sum_{j=0}^{m-1} \frac{1}{j !} K_{m-1}^{(j)}\left(1-\Lambda ;|x|^{2}-1\right) q_{j}(0)=v_{1}(x)+v_{0}(x),
\end{aligned}
$$

where $v_{0}(x)$ is the $m$-harmonic polynomial. Now, it is necessary to solve the equation $\Lambda u=v$ in $m$-harmonic functions in $S$. The functions $v_{i}(x)$ are $m$-harmonic by construction. Note that for any $m$-harmonic in $S$ function $p(x)$, the equality $(\Lambda p)(0)=0$ is true, and therefore, $v_{1}(0)=0$. Find the value $v_{0}(0)$. It is not hard to see that

$$
v_{0}(0)=\sum_{j=0}^{m-1} \frac{q_{j}(0)}{j !} K_{m-1}^{(j)}(1 ;-1)=\sum_{j=0}^{m-1} \frac{q_{j}(0)}{j !}\left(\sum_{k=0}^{m-1}(-1)^{k} H_{k}(\lambda)\right)^{(j)}(1) .
$$

Lemma 5. Let, in accordance with (27)

$$
H_{k}(\lambda)=\frac{1}{(2 k) ! !} \lambda(\lambda-2) \cdots(\lambda-2 k+2), k \in \mathbb{N} ; \quad H_{0}(\lambda)=1 .
$$

Then, for $j \in \mathbb{N}_{0}$, the following equality holds true:

$$
\left(\sum_{k=0}^{m-1}(-1)^{k} H_{k}(\lambda)\right)^{(j)}(1)=(-1)^{m-1} \frac{2 m}{j+1} H_{m}^{(j+1)}(0) .
$$

Proof. Let us prove the equality (43) by the method of mathematical induction on $m$. For $m=0$, we have zeros on the left-hand and on the right-hand sides of (43), and for $m=1$, we also have the equality $H_{0}^{(j)}(1)=\frac{2}{j+1} H_{1}^{(j+1)}(1)$, where $j \in \mathbb{N}_{0}$. Under the assumption that the equality (43) is true for $m \geq 1$, we prove its validity for $m=m+1$. According to the definition of the derivative (13), we have $(f(\lambda) g(\lambda))^{(1)}=f^{(1)}(\lambda) g(\lambda+1)+f(\lambda) g^{(1)}(\lambda)$, and therefore, taking into account that $H_{m+1}(\lambda)=H_{m}(\lambda)(\lambda-2 m) /(2 m+2)$, we write

$$
H_{m+1}^{(1)}(\lambda)=\frac{1}{2 m+2}\left(H_{m}^{(1)}(\lambda)(\lambda-2 m+1)+H_{m}(\lambda)\right),
$$

whence

$$
H_{m+1}^{(2)}(\lambda)=\frac{1}{2 m+2}\left(H_{m}^{(2)}(\lambda)(\lambda-2 m+2)+2 H_{m}^{(1)}(\lambda)\right)
$$

and therefore,

$$
H_{m+1}^{(j+1)}(\lambda)=\frac{1}{2 m+2}\left(H_{m}^{(j+1)}(\lambda)(\lambda-2 m+j+1)+(j+1) H_{m}^{(j)}(\lambda)\right) .
$$

Thus, the right-hand side of (43) for $m=m+1$ is transformed to the form

$$
\begin{gathered}
(-1)^{m} \frac{2 m+2}{j+1} H_{m+1}^{(j+1)}(0)=\frac{(-1)^{m}}{j+1}\left(H_{m}^{(j+1)}(0)(-2 m+j+1)+(j+1) H_{m}^{(j)}(0)\right) \\
=(-1)^{m-1} \frac{2 m}{j+1} H_{m}^{(j+1)}(0)+(-1)^{m}\left(H_{m}^{(j+1)}(0)+H_{m}^{(j)}(0)\right)= \\
=\sum_{k=0}^{m-1}(-1)^{k} H_{k}^{(j)}(1)+(-1)^{m} H_{m}^{(j)}(1)=\sum_{k=0}^{m}(-1)^{k} H_{k}^{(j)}(1)
\end{gathered}
$$


which proves the induction step. Here, in the second line, the induction hypothesis is used. The lemma is proved.

If, in (42), we use the just-proved lemma, the equality $H_{m}(\lambda)=h_{m}(\lambda) /(2 m) ! !$, and the condition (39), then we get

$$
\begin{array}{rl}
v_{0}(0)=(-1)^{m-1} 2 & m \sum_{j=0}^{m-1} q_{j}(0) \frac{H_{m}^{(j+1)}(0)}{(j+1) !} \\
& =\frac{(-1)^{m-1}}{(2 m-2) ! !} \sum_{j=0}^{m-1} q_{j}(0) \frac{h_{m}^{(j+1)}(0)}{(j+1) !}=\frac{(-1)^{m-1}}{(2 m-2) ! !} \sum_{j=0}^{m-1} q_{j}(0) h_{m}^{(j+1)}=0 .
\end{array}
$$

The converse is also true, that is, $v(0)=0 \Rightarrow v_{0}(0)=0 \Rightarrow$ (39). In [15], it is proved that the equation $\Lambda u=v$ is solvable in $m$-harmonic functions only if $v(0)=$ 0 , but this condition is satisfied. The solution to the equation $\Lambda u=v$ can be written as (40) $u(x)=\int_{0}^{1} v(t x) \frac{d t}{t}+C$. With smoothness imposed on the boundary functions $\psi_{k} \in$ $C^{2 m-1-k+\varepsilon}(\partial S), k=0, \ldots, m-1$, similarly to the Theorem 4 , we have $q_{k} \in C^{2 m-1-k}(\partial S)$, and hence, since in the formula (32) the differential operator applied to the function $q_{k}$ has the order $m-1-k$, then $v=\Lambda u \in C^{m}(\bar{S})$, and by $(40) u \in C^{m}(\bar{S})$. Thus, the $m$-harmonic function (40) is a solution to the Neumann boundary value problem (5)-(6). If a solution to the Neumann boundary value problem $u(x)$ exists, then the function $v(x)$ from (41) must satisfy the equality $\Lambda u=v$, which is possible only if $v(0)=0$, whence follows equality (39). The solution $u(x)$ is unique up to a constant. The theorem is proved.

Remark 4. The solvability condition for the Neumann boundary value problem (39) obtained in Theorem 6 does not impose an explicit condition on the boundary functions $\psi_{k}$, but only connects the values of harmonic functions $q_{k}(0), k=0, \ldots, m-1$. This condition does not formally coincide with the solvability condition (23) from Remark 2.

Lemma 6. The solvability condition (39) and the condition from Remark 2 are equivalent.

Proof. First, we rewrite the condition (23) for the $m$-harmonic equation and the Neumann boundary conditions taken in the form $\left.\frac{\partial j u}{\partial v^{j}}\right|_{\partial S}=\psi_{j}, j=0, \ldots, m-1$

$$
0=\int_{\partial S} \sum_{j=1}^{m} h_{m+1, m}^{(j)} \psi_{j-1} d s_{\xi}=\int_{\partial S} \sum_{j=0}^{m-1} h_{m+1, m}^{(j+1)} \psi_{j} d s_{\xi},
$$

where $h_{m+1, m}(\lambda)$ are elements of the last row of the matrix $\mathbb{H}_{m+1}$. If we notice that $h_{m+1, m}(\lambda)=h_{m}(\lambda) /(2 m) !$ ! and take into account that for the harmonic functions $q_{j}(x)$, the equality $\frac{1}{\omega_{n}} \int_{\partial S} \psi_{j} d s_{\xi}=q_{j}(0)$ holds, then the condition (23) for the $m$-harmonic equation takes the form

$$
0=\frac{1}{(2 m) ! !} \int_{\partial S} \sum_{j=0}^{m-1} h_{m}^{(j+1)} \psi_{j} d s_{\xi}=\frac{\omega_{n}}{(2 m) ! !} \sum_{j=0}^{m-1} h_{m}^{(j+1)} q_{j}(0) .
$$

This condition is equivalent to the condition (39). The lemma is proved.

Example 4. Let us find a solution to the Neumann boundary value problem (5) and (6) for the three-harmonic equation in the unit ball $(m=3)$. In accordance with the method of constructing a solution to the Neumann boundary value problem from Theorem 6, we use the solution to the Dirichlet boundary value problem from Example 2 and replace, in this solution, the operator $\Lambda$ by the operator $\Lambda-1$. After simple transformations, we get 


$$
\begin{aligned}
v(x)=\sum_{j=0}^{2} \frac{1}{j !} K_{\lambda}^{(j)}\left(1-\Lambda ;|x|^{2}-1\right) q_{j}(x) & =\left(1+\frac{1}{2}\left(|x|^{2}-1\right)-\frac{1}{4 ! !}\left(|x|^{2}-1\right)^{2}\right. \\
\left.-\frac{1}{2}\left(|x|^{2}-1\right) \Lambda+\frac{1}{4 ! !}\left(|x|^{2}-1\right)^{2} \Lambda^{2}\right) q_{0}(x)+\left(\frac{1}{2}\left(|x|^{2}-1\right)+\frac{1}{4 ! !}\left(|x|^{2}-1\right)^{2}\right. & \left.-\frac{2}{4 ! !}\left(|x|^{2}-1\right)^{2} \Lambda\right) q_{1}(x)+\frac{1}{4 ! !}\left(|x|^{2}-1\right)^{2} q_{2}(x) .
\end{aligned}
$$

Find $v(0)$. It is clear that $v(0)=\frac{1}{4 ! !}\left(3 q_{0}(0)-3 q_{1}(0)+q_{2}(0)\right)$. Since $h_{3}(\lambda)=\lambda(\lambda-2)(\lambda-4)=3 \lambda^{[1]}-3 \lambda^{[2]}+\lambda^{[3]}$, then the condition (39) has the form

$$
\sum_{j=0}^{2} h_{3}^{(j+1)} q_{j}(0)=3 q_{0}(0)-3 q_{1}(0)+q_{2}(0)=0,
$$

which corresponds to the condition $v(0)=0$. Under this condition, the solution (40) to the Neumann boundary value problem has the form

$$
\begin{aligned}
u(x)=\int_{0}^{1} v(t x) \frac{d t}{t}= & \int_{0}^{1}\left(\left(1+\frac{1}{2}\left(|x|^{2} t^{2}-1\right)-\frac{1}{4 ! !}\left(|x|^{2} t^{2}-1\right)^{2}\right.\right. \\
-\frac{1}{2}\left(|x|^{2} t^{2}-1\right) \Lambda+ & \left.\frac{1}{4 ! !}\left(|x|^{2} t^{2}-1\right)^{2} \Lambda^{2}\right) q_{0}(t x)+\left(\frac{1}{2}\left(|x|^{2} t^{2}-1\right)+\frac{1}{4 ! !}\left(|x|^{2} t^{2}-1\right)^{2}\right. \\
& \left.\left.\quad-\frac{2}{4 ! !}\left(|x|^{2} t^{2}-1\right)^{2} \Lambda\right) q_{1}(t x)+\frac{1}{4 ! !}\left(|x|^{2} t^{2}-1\right)^{2} q_{2}(t x)\right) \frac{d t}{t}+C .
\end{aligned}
$$

In the particular case when $\psi_{i}, i=0,1,2$ are constant, we have $q_{i}(x)=\psi_{i}$ and

$$
u(x)=\frac{|x|^{2}}{8}\left(3 \psi_{0}+\psi_{1}-\psi_{2}\right)+\frac{|x|^{4}}{32}\left(-\psi_{0}+\psi_{1}+\psi_{2}\right)+C,
$$

provided that $3 \psi_{0}-3 \psi_{1}+\psi_{2}=0$.

\section{Conclusions}

It is well-known (see [37]) that, if a function $u$ is $m$-harmonic in a star domain, then it can be represented by Almansi's expansion $u(x)=\sum_{k=0}^{m-1} p_{k}(x)|x|^{2 k}$, where $p_{k}(x)$, $k=0, \ldots, m-1$ are harmonic functions. In the present work, we managed to slightly transform this expansion and find the functions $p_{k}(x)$ in an explicit form for solving the Dirichlet and Neumann problems in the unit ball. As far as we know, such a representation of solutions of boundary value problems for elliptic equations of arbitrary order $2 m$ is obtained for the first time.

The representations of solutions of the Dirichlet and Neumann problems obtained in Theorems 4 and 6 have advantages over formulas like (1) using the Green's function of the problem, in their relative simplicity (no need to calculate singular integrals of Green's function) and transparency (the dependence of the solution to the problem on each of the boundary functions is visible).

The proposed method can be used to represent solutions of other types of boundary value problems for the polyharmonic equation, and can also be used when applying the Adomian decomposition method for nonlinear boundary value problems. The presented method is also useful for constructing solutions to model boundary value problems for computational purposes. As an extension of the results obtained to other differential equations, we can mark an equation of the form $\lambda_{1} u_{x_{1} x_{1}}+\ldots+\lambda_{n} u_{x_{n} x_{n}}=0$, since an analogue of the Almansi formula is known for it [35].

Funding: The work was supported by Act 211 of the Government of the Russian Federation, contract no. 02.A03.21.0011.

Data Availability Statement: Not applicable. 
Conflicts of Interest: The author declares no conflict of interest.

\section{References}

1. Begehr, H. Biharmonic Green functions. Matematiche 2006, 61, 395-405.

2. Karachik, V.V.; Turmetov, B.K. On Green's function of the Robin problem for the Poisson equation. Adv. Pure Appl. Math. 2019, 10, 203-214. [CrossRef]

3. Karachik, V. Green's function of Dirichlet problem for biharmonic equation in the ball. Complex Var. Elliptic Equ. 2019, 64, 1500-1521. [CrossRef]

4. Karachik, V.V. The Green Function of the Dirichlet Problem for the Triharmonic Equation in the Ball. Math. Notes 2020, 107, 105-120. [CrossRef]

5. Wang, Y.; Ye, L. Biharmonic Green function and biharmonic Neumann function in a sector. Complex Var. Elliptic Equ. 2013, 58, 7-22. [CrossRef]

6. Wang, Y. Tri-harmonic boundary-value problems in a sector. Complex Var. Elliptic Equ. 2014, 59, 732-749. [CrossRef]

7. Constantin, E.; Pavel, N.H. Green function of the Laplacian for the Neumann problem in $\mathbb{R}_{+}^{n}$. Lib. Math. 2010, 30, 57-69.

8. Begehr, H.; Vaitekhovich, T. Modified harmonic Robin function. Complex Var. Elliptic Equ. 2013, 58, 483-496. [CrossRef]

9. Gazzola, F.; Grunau, H.C.; Sweers, G. Polyharmonic Boundary Value Problems: Positivity Preserving and Nonlinear Higher Order Elliptic Equations in Bounded Domains; Springer: Berlin, Germany, 1991.

10. Boggio, T. Sulle funzioni di Green dórdine m. Rend. Circ. Mat. Palermo 1905, 20, 97-135. [CrossRef]

11. Begerh, H.; Vu, T.N.H.; Zhang, Z.-X. Polyharmonic Dirichlet Problems. Proc. Steklov Inst. Math. 2006, $255,13-34$.

12. Kal'menov, T.S.; Suragan, D. On a new method for constructing the Green function of the Dirichlet problem for the polyharmonic equation. Differ. Equ. 2012, 48, 441-445. [CrossRef]

13. Karachik, V.V. Construction of polynomial solutions to the Dirichlet problem for the polyharmonic equation in a ball. Comput. Math. Math. Phys. 2014, 54, 1122-1143. [CrossRef]

14. Karachik, V.V. Generalized Third Boundary Value Problem for the Biharmonic Equation. Differ. Equ. 2017, 53, 756-765. [CrossRef]

15. Karachik, V.V. A Neumann-type problem for the biharmonic equation. Sib. Adv. Math. 2017, 27, 103-118. [CrossRef]

16. Karachik, V.V. Riquier-Neumann Problem for the Polyharmonic Equation in a Ball. Differ. Equ. 2018, 54, 648-657. [CrossRef]

17. Koshanov, B.D.; Soldatov, A.P. Boundary value problem with normal derivatives for a high-order elliptic equation on the plane. Differ. Equ. 2016, 52, 1594-1609. [CrossRef]

18. Begehr, H.; Burgumbayeva, S.; Shupeyeva, B. Green and Neumann Functions for a Plane Degenerate Circular Domain. Trends Math. 2019, 141-149._13. [CrossRef]

19. Begehr, H.; Burgumbayeva, S.; Shupeyeva, B. Remark on Robin problem for Poisson equation. Complex Var. Elliptic Equ. 2017, 62, 1589-1599. [CrossRef]

20. Akel, M.; Begehr, H. Neumann function for a hyperbolic strip and a class of related plane domains. Math. Nachrichten 2017, 290, 490-506. [CrossRef]

21. Lin, H. Harmonic Green and Neumann functions for domains bounded by two intersecting circular arcs. Complex Var. Elliptic Equ. 2020, doi:10.1080/17476933.2020.1816984. [CrossRef]

22. Begehr, H.; Burgumbayeva, S.; Dauletkulova, A.; Lin, H. Harmonic Green functions for the Almaty apple. Complex Var. Elliptic Equ. 2020, 65, 1814-1825. [CrossRef]

23. Dong, H.; Li, H. Optimal Estimates for the Conductivity Problem by Green's Function Method. Arch. Ration. Mech. Anal. 2019, 231, 1427-1453. [CrossRef]

24. Grebenkov, D.S.; Traytak, S.D. Semi-analytical computation of Laplacian Green functions in three-dimensional domains with disconnected spherical boundaries. J. Comput. Phys. 2019, 379, 91-117. [CrossRef]

25. Hsu, C.-W.; Hwu, C. Green's functions for unsymmetric composite laminates with inclusions. Proc. R. Soc. A Math. Phys. Eng. Sci. 2020, 476, 20190437. [CrossRef]

26. Van Dokkum, J.S.; Nicola, L. Green's function molecular dynamics including viscoelasticity. Model. Simul. Mater. Sci. Eng. 2019, 27, 075006. [CrossRef]

27. Karachik, V.V. Green's Functions of the Navier and Riquier-Neumann Problems for the Biharmonic Equation in the Ball. Differ. Equ. 2021, 57, 654-668. [CrossRef]

28. Karachik, V.V. Presentation of solution of the dirichlet problem for bigharmonic equation in the unit ball through the Green function. Chelyabinsk Phys. Math. J. 2020, 5, 391-399. [CrossRef]

29. Koshlyakov, N.S.; Gliner, E.B.; Smirnov, M.M. Differential Equations of Mathematical Physics; North-Holland: Amsterdam, The Netherlands, 1964. [CrossRef]

30. Karachik, V.V.; Antropova, N.A. On the solution of the inhomogeneous polyharmonic equation and the inhomogeneous Helmholtz equation. Differ. Equ. 2010, 46, 387-399. [CrossRef]

31. Bateman, H. Higher Transcendental Functions; Bateman Manuscript Project; Erdelyi, A., Ed.; McGraw-Hill: New York, NY, USA, 1953. [CrossRef]

32. Karachik, V.V. On the mean value property for polyharmonic functions in the ball. Sib. Adv. Math. 2014, 24, 169-182. [CrossRef]

33. Karachik, V.V. On the Arithmetic Triangle Arising from the Solvability Conditions for the Neumann Problem. Math. Notes 2014, 96, 217-227. [CrossRef] [PubMed] 
34. Almansi, E. Sull' integrazione dell' equazione differenziale $\Delta^{n} u=0$. Ann. Mat. Pura Appl. 1899, 11, 5-19. [CrossRef]

35. Karachik, V.V. On an expansion of Almansi type. Math. Notes 2008, 83, 335-344. [CrossRef]

36. Alimov, S.A. On a problem with an oblique derivative. Differ. Equ. 1981, 17, 1738-1751. [CrossRef]

37. Sobolev, S.L. Cubature Formulas and Modern Analysis: An Introduction; Nauka: Moscow, Russia, 1974; Reprint in Gordon and Breach: Montreux, Switzerland, 1992. [CrossRef] 